TecnoHumanismo. Revista Científica

Junio - Agosto 2021

Volumen 1 / No.2

ISSN: $2710-2394$

pp. $145-163$

https://doi.org/10.53673/th.v1i9.56

https://tecnohumanismo.online

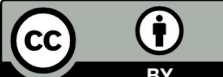

\title{
Caracterización y evaluación de potencia y eficiencia de un sistema híbrido eolico fotovoltaico off-grid
}

\author{
Characterization and evaluation of power and efficiency of a hybrid wind photovoltaic off-grid system
}

Caracterização e avaliação da potência e eficiência de um sistema híbrido eólico fotovoltaico fora da rede

\section{ARTÍCULO ORIGINAL}

Anapaula Marianella Solis Alberca

asolisalberca@,gmail.com

https://orcid.org/0000-0003-2421-6183

Universidad Nacional del Santa, Ancash - Perú

\author{
Denis Javier Aranguri Cayetano \\ aranguricdj@.gmail.com \\ https://orcid.org/0000-0002-6119-2072 \\ Universidad Nacional del Santa, \\ Ancash - Perú
}

\author{
Ronny Jhonatan Sáenz Gutiérrez \\ ronysgutierrez@outlook.es \\ https://orcid.org/0000-0003-4197-0153 \\ Universidad Nacional del Santa, \\ Ancash - Perú
}

Recibido 05 de Febrero 2021 | Arbitrado y aceptado 19 de Abril 2021 | Publicado 28 de Julio 2021

\section{RESUMEN}

La diversificación de la matriz energética es un ámbito fundamental para el desarrollo del Perú, lo cual conlleva al desarrollo de energía sustentable y renovable, y que a su vez reduzcan al mínimo los potenciales impactos negativos ambientales; siendo las opciones más viables el recurso eólico y solar en función de nuestra situación geográfica. Es así, que el presente artículo presenta el estudio de tecnología híbrida autosustentable, mediante la presentación de datos a tiempo real obtenidos en el Módulo Solar-Eólico Energy Cube instalado en la Universidad Nacional del Santa, Nuevo Chimbote/ Ancash, con una potencia nominal de $6200 \mathrm{Wp}$. $\mathrm{Se}$ ha realizado el estudio del rendimiento de generación de energía eléctrica y la eficiencia de conversión de energía, por la disposición del recurso eólico-solar en función de la potencia generada tanto por los módulos fotovoltaicos, así como por el aerogenerador, siendo caracterizado como un sistema conjunto.

En función al periodo de funcionamiento evaluado, los resultados obtenidos indican que la potencia máxima obtenida fue el 14 de octubre equivalente a $1896.3 \mathrm{~W}$ con un rendimiento de generación de energía eléctrica promedio de $30.58 \%$ y una eficiencia de conversión promedio de $13.37 \%$ con datos meteorológicos de irradiación solar promedio de $764.67 \mathrm{~W} / \mathrm{m}^{\wedge} 2$ y una velocidad de viento promedio de $5 \mathrm{~m} / \mathrm{s}$, alcanzando un máximo de rendimiento de generación de $55.48 \%$ a las 11:45 de la mañana; y la potencia mínima obtenida fue el 13 de junio equivalente a $333.8 \mathrm{~W}$ con rendimiento del sistema y eficiencia de conversión promedio del sistema de $8.09 \%$ y $7.5 \%$ respectivamente, con datos meteorológicos promedios de irradiación solar de $196.6 \mathrm{~W} / \mathrm{m}^{\wedge} 2$ y velocidad de viento de $1.2 \mathrm{~m} / \mathrm{s}$.

Palabras-clave: Energía fotovoltaica, energía eólica, sistema híbrido, rendimiento.

\section{ABSTRACT}

The diversification of the energy matrix is a fundamental area for the development of Peru, which leads to the development of sustainable and renewable energy, and which in turn reduces the potential negative environmental impacts to a minimum; the most viable options being wind and solar resources depending on our geographical location. Thus, this article presents the study of selfsustaining hybrid technology, through the presentation of real-time data obtained in the SolarWind Energy Cube Module installed at the Universidad Nacional del Santa, Nuevo Chimbote / Ancash, with a nominal power of $6200 \mathrm{Wp}$.

A study has been carried out of the performance of electric power generation and the efficiency of energy conversion, by the disposition of the windsolar resource based on the power generated by both the photovoltaic modules, as well as by the wind turbine, being characterized as a joint system.

Based on the evaluated operating period, the results obtained indicate that the maximum power obtained on October 14 was equivalent to $1896.3 \mathrm{~W}$ with an average electrical power generation performance of $30.58 \%$ and an average conversion efficiency of $13.37 \%$ with meteorological data. average solar irradiation of $764.67 \mathrm{~W} / \mathrm{m}^{\wedge} 2$ and an average wind speed of $5 \mathrm{~m} / \mathrm{s}$, reaching a maximum generation performance of $55.48 \%$ at $11: 45$ in the morning; and the minimum power obtained was on June 13 equivalent to $333.8 \mathrm{~W}$ with system performance and average conversion efficiency of the system of $8.09 \%$ and $7.5 \%$ respectively, with average meteorological data of solar irradiation of $196.6 \mathrm{~W}$ $/ \mathrm{m}^{\wedge} 2$ and speed of $1.2 \mathrm{~m} / \mathrm{s}$ wind.

Keywords: Photovoltaic energy, wind energy, hybrid system, performance.

\section{RESUMO}

A diversificação da matriz energética é uma área fundamental para o desenvolvimento do Peru, o que leva ao desenvolvimento de energia sustentável e renovável, e que por sua vez reduz ao mínimo os potenciais impactos ambientais negativos; as opções mais viáveis são os recursos eólicos e solares, dependendo da nossa localização geográfica. Assim, este artigo apresenta o estudo da tecnologia híbrida autossustentável, por meio da apresentação de dados em tempo real obtidos no Módulo Cubo de Energia Solar-Eólica instalado na Universidade Nacional del Santa, Nuevo Chimbote / Ancash, com potência nominal de $6200 \mathrm{Wp}$.

Foi realizado um estudo do desempenho da geração de energia elétrica e da eficiência de conversão de energia, pela disposição do recurso eólico-solar com base na potência gerada tanto pelos módulos fotovoltaicos, quanto pelo aerogerador, sendo caracterizado como um sistema conjunto.

Com base no período operacional avaliado, os resultados obtidos indicam que a potência máxima obtida em 14 de outubro foi equivalente a $1896,3 \mathrm{~W}$ com um desempenho médio de geração de energia elétrica de $30,58 \%$ e uma eficiência de conversão média de $13,37 \%$ com dados meteorológicos. Irradiação solar média de $764,67 \mathrm{~W} / \mathrm{m}^{\wedge} 2$ e velocidade média do vento de $5 \mathrm{~m} / \mathrm{s}$, atingindo desempenho máximo de geração de $55,48 \%$ às $11 \mathrm{~h} 45$ da manhã; e a potência mínima obtida foi no dia 13 de junho equivalente a $333,8 \mathrm{~W}$ com desempenho do sistema e eficiência média de conversão do sistema de $8,09 \%$ e $7,5 \%$ respectivamente, com dados meteorológicos médios de irradiação solar de $196,6 \mathrm{~W} / \mathrm{m}^{\wedge} 2$ e velocidade de $1,2 \mathrm{~m} / \mathrm{s}$ vento.

Palavras-chave: Energia fotovoltaica, energia eólica, sistema híbrido, desempenho. 


\section{INTRODUCCION}

En el Perú, la generación eléctrica se ha encontrado históricamente concentrada en los recursos hídricos y térmicos (mediante la quema de combustibles). Sin embargo, la excesiva cantidad de gases contaminantes producidos por las centrales térmicas y el impacto ambiental que conlleva la construcción de centrales que operan con fuentes hídricas ha provocado el impulso de la exploración de las fuentes renovables en la generación de energía eléctrica buscando ocasionar un impacto ambiental positivo. En 2008, mediante el Decreto Legislativo $N^{\circ}$ 1002, Ley de Promoción de la Inversión en Generación de Electricidad con el uso de Energías Renovables, se estableció la promoción de este tipo de fuentes de energía para mejorar la calidad de vida de la población y proteger el ambiente. (OSINERGMIN, 2017).

Las energías renovables tienen numerosas ventajas, pero también presentan ciertos desafíos importantes al sistema de suministro. Acorde con Carrera y Diéguez (2021) una de las características más exigentes es la variabilidad incontrolable de la fuente. No es posible garantizar las condiciones ambientales con anticipación y, en consecuencia, tampoco el suministro. Este problema se puede solventar almacenando energía durante épocas de generación de potencia excesiva y utilizándola cuando la generación de potencia es moderada.

El avance tecnológico ha demostrado que los recursos renovables pueden ser utilizados paralelamente mediante sistemas denominados "híbridos". Estos sistemas, según explican Ramos y Luna (2014) pueden generar energía eléctrica a partir de dos o más fuentes de energía renovables simultáneamente, tal es el caso particular del sistema en estudio, eólico y fotovoltaico.

En una investigación reciente (González, 2019), señala que un sistema híbrido optimiza las mejores condiciones del viento y del sol, completamente entre sí. Los días fríos y de viento, normalmente nubosos, apenas permiten aprovechar la luz solar, mientras que son ideales para los aerogeneradores. Por su parte, los días de cielos despejados con poco viento, son los más adecuados para los paneles fotovoltaicos

El escenario de la investigación está dado en el EDS Energy Cube (Environmental Discovery System) instalado en la Universidad Nacional del Santa, el cual es un sistema independiente con un fluctuante rendimiento de los productores de energía, es decir, los generadores de energía solares y una turbina de energía eólica. 
En este artículo de investigación se recolectó y analizó el potencial eólico-solar mediante instrumentación y obtención de datos reales para cuantificar la cantidad de energía que genera el sistema híbrido eólico- fotovoltaico.

De la misma manera, en una investigación realizada, por (Rojas, 2012), en la Universidad Nacional del Santa, las velocidades de viento a una altura de $12 \mathrm{~m}$ alcanzaron valores promedios de $5 \mathrm{~m} / \mathrm{s}$, con una velocidad menor de $1 \mathrm{~m} / \mathrm{s}$ y una velocidad máxima de $9 \mathrm{~m} / \mathrm{s}$, dirección de viento predominante sureste.

Actualmente se proyectan sistemas híbridos en los que las fuentes renovables y el almacenamiento proporcionan hasta un 80-90 \% de la necesidad energética (Ruiz, 2019). Según, Mitaki et al. (2012), la instalación de recursos de pequeña escala se está extendiendo sin un estudio serio de eficiencia y viabilidad, debido a gran parte a que no se cuentan con modelos que permitan hacer un análisis y predicciones sobre su utilidad real.

De acuerdo con la fuente previa, es muy común que los sistemas de suministro de energías renovables tengan algún aporte auxiliar como un generador eléctrico de Diesel o una conexión a la red eléctrica. Si ya existe la conexión, la red eléctrica suele ser la fuente auxiliar más económica.

Sistema híbrido eólico fotovoltaico On-grid. Es un sistema de generación de energía renovable que permite el trabajo en paralelo con la red de energía eléctrica pública. En este caso no hace falta el almacenamiento, sino que el mismo sistema de suministro renovable interacciona con la red siempre y cuando la demanda no sea igual al suministro renovable, pudiendo incluso inyectar directamente a la red mediante un inversor.

Sistema híbrido eólico fotovoltaico Off-grid. Es un sistema de generación de energía renovable que puede estar conectado a un sistema de contingencia, como la red eléctrica, cuando el consumo es mayor que la generación de energía, mas no puede inyectar a la red. También existen caso donde si no está conectado a un sistema auxiliar simplemente se apaga a la espera de que recurso sea nuevamente viable.

\section{DESCRIPCION DEL SISTEMA HÍBRIDO EÓLICO FOTOVOLTAICO}

Son aquellos sistemas fotovoltaicos que se apoyan de otra fuente generadora secundaria que pertenece a otro sistema de generación eléctrica por ejemplo eólica (Villamil y 
Villamil, 2019). En este caso, el sistema híbrido eólico fotovoltaico fue instalado en

Setiembre del 2017 y está ubicado en la loza solar de la escuela de Ingeniería en Energía de la Universidad Nacional del Santa constituido por:

Tabla 1. Elementos numerados del sistema de energía eólica y solar del sistema híbrido.

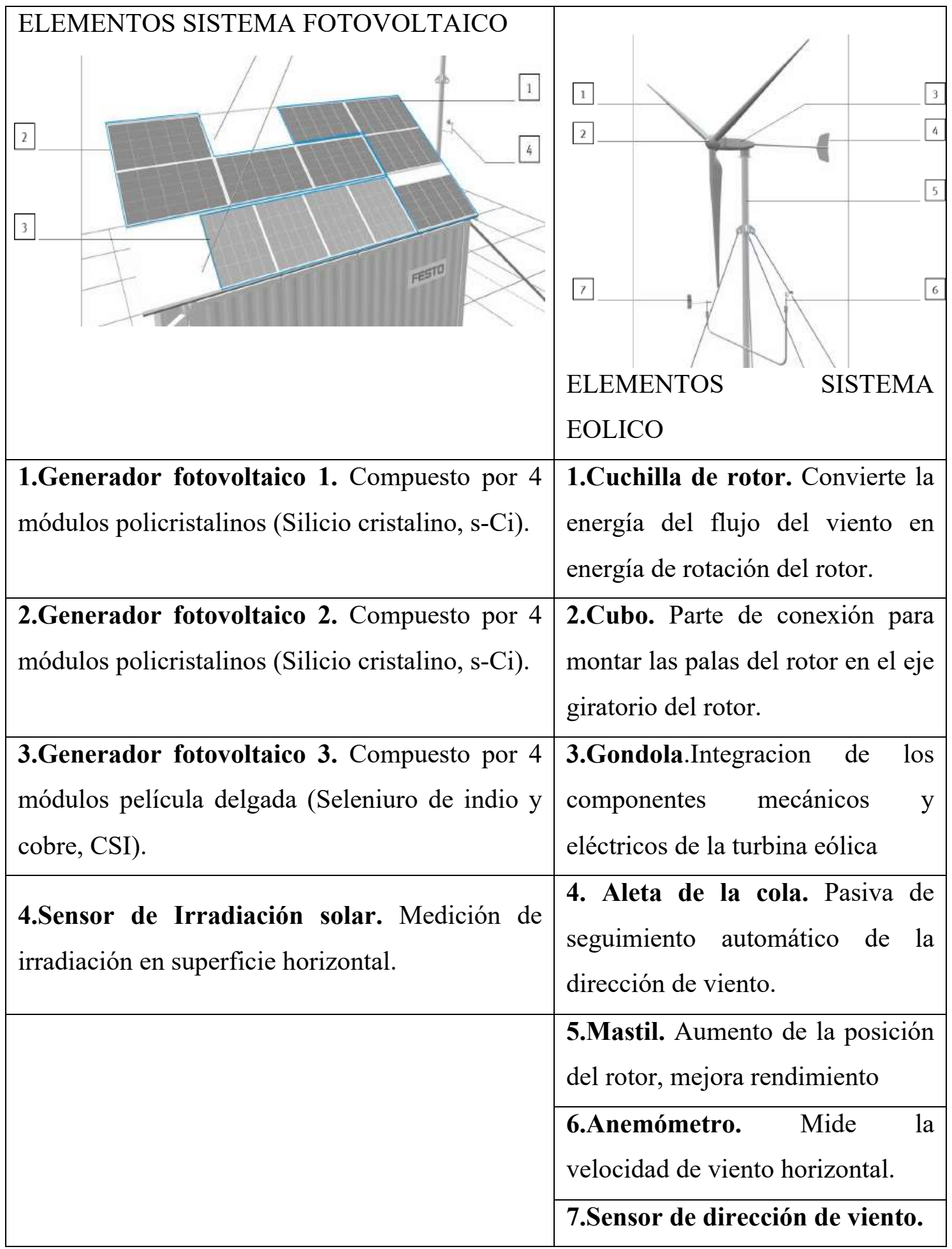


Doce módulos fotovoltaicos que cubren una superficie total de $18 \mathrm{~m}^{2}$ y una capacidad instalada de $2.7 \mathrm{kWp}$. Cuenta con la tecnología de 02 grupos de módulos fotovoltaicos de $1 \mathrm{~kW}$ con una eficiencia nominal de $15.28 \%$ de marca Astro Energy y 01 grupo de módulos de película delgada CIS sobre sustrato de vidrio de marca Solar Frontier de 0.7 $\mathrm{kW}$ de potencia con una eficiencia nominal de $13.8 \%$. Las eficiencias y parámetros operativos de los módulos están medidos en condiciones de prueba estándar "STC", definidas por la norma IEC $\left(1000 \mathrm{~W} / \mathrm{m}^{2}\right.$ de irradiación, $25^{\circ} \mathrm{C}$ de temperatura y a una densidad espectral de AM 1,5), estando conectados en serie.

Cuenta con una turbina eólica de marca Antaris instalado a $15 \mathrm{~m}$ por encima del suelo y una capacidad instalada de $3.5 \mathrm{kWp}$. Esta específicamente diseñado para el suministro de la red, de la batería (24 VDC y 48 VDC) y diseñado para soportar sistemas de calefacción. Como resultado de un diseño robusto, este aerogenerador también es adecuado para sitios de viento expuesto, con un diámetro de rotor de $3.5 \mathrm{~m}$.

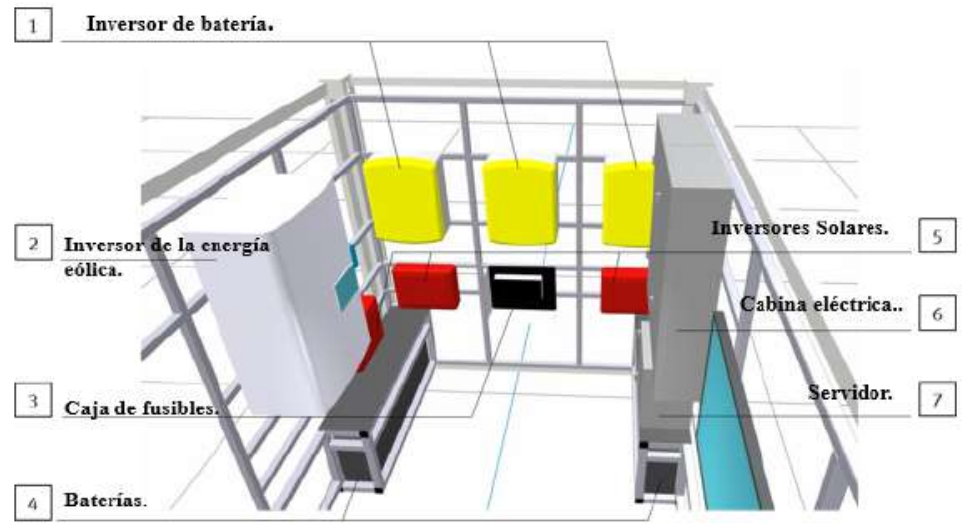

Figura. 1. Componentes dentro del EDS Energy Cube (Environmental Discovery System)

El inversor fotovoltaico es de marca SMA, Sunny Boy, tiene una amplia zona de tensión de entrada que comprende desde los 80 hasta los 600 V. El Sunny Boy es un inversor fotovoltaico sin transformador que convierte la corriente continua del generador fotovoltaico en corriente alterna compatible con la red, siendo capaz de inyectar a la red de suministro eléctrico.

El Sunny Island es un inversor de batería que controla el equilibrio de energía eléctrica en un sistema fuera de la red. Es un sistema de respaldo por baterías o en un sistema de un mayor autoconsumo. Es perfecta para sistemas independientes o conectados a la red con un rango de potencia de salida de 2 a 13kW. Con su clase de protección alta, amplio 
rango de temperatura y capacidad de sobrecarga, el Sunny Island proporciona el tipo de confiabilidad necesaria para el uso fuera de la red. La gestión inteligente de la carga y la energía garantiza el funcionamiento incluso en situaciones críticas, como en el caso de altas corrientes de entrada o condiciones ambientales adversas.

Tiene un convertidor de alimentación Smart Wind que trabaja con la pequeña turbina eólica Antaris de $3.5 \mathrm{~kW}$ de potencia que garantiza un arranque adecuado de la pequeña turbina eólica e incluso controla cualquier otro estado de condición en el mejor de los casos. Su control de curva característica inteligente conduce a una óptima explotación del viento. El dispositivo monitorea el generador, la turbina eólica y a la red eléctrica, llevando incluso la turbina eólica a un estado seguro en caso de error. El monitoreo de la velocidad del generador y el de la temperatura del generador están disponibles de manera estándar en su rango de operación.

Todos estos componentes fueron diseñados especialmente para plantas de generación fotovoltaica y eólica pequeñas para usar su potencial al máximo.

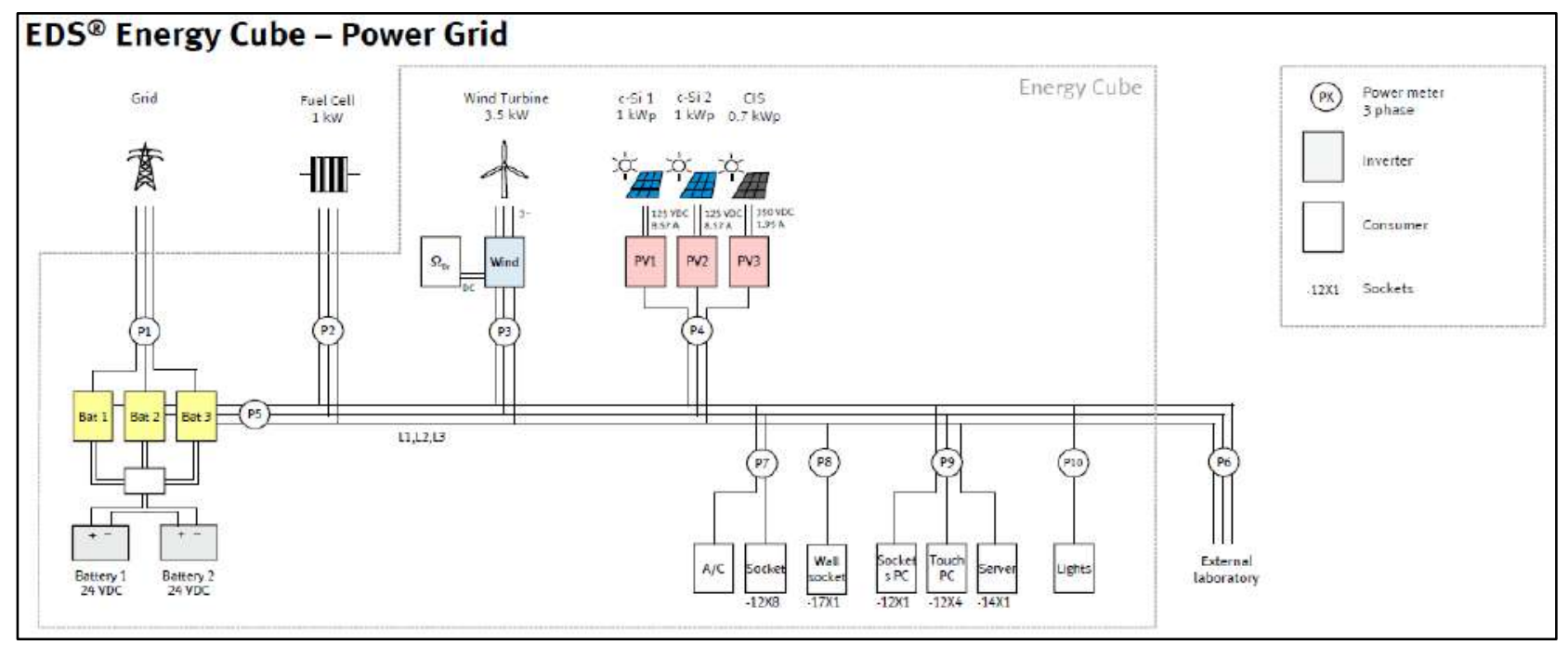

Figura. 2. Diagrama Unifilar de los elementos que conforman sistema híbrido eólico fotovoltaico. Inversor fotovoltaico Sunny Boy (rojos), Inversores Sunny Island (amarillos), inversor eólico (celeste).

Tabla 2. Especificaciones técnicas de los módulos fotovoltaicos del sistema híbrido para las condiciones de irradiancia $1000 \mathrm{~W} / \mathrm{m}^{2}$, temperatura de célula $25{ }^{\circ} \mathrm{C}$ y espectro $\mathrm{AM}$ 1,5 . 
Módulo Fotovoltaico

Potencia nominal $\left(P_{\max }\right)$

Tolerancia de potencia

Eficiencia del módulo

Voltaje de Circuito abierto $\left(V_{o c}\right)$

Corriente de Corto circuito $\left(I_{s c}\right)$

Tensión nominal $\left(V_{m p p}\right)$

Corriente Nominal $\left(I_{m p p}\right)$

Coeficiente $\alpha$

Coeficiente $\beta$

Coeficiente $\delta$

Área

\begin{tabular}{|l|l|l|}
$\begin{array}{l}\text { Silicio } \\
\text { policristalino }\end{array}$ & $\begin{array}{l}\text { Película } \\
\text { delgada } \\
\text { CSI }\end{array}$ & Unidad \\
\hline 250 & 175 & Watts \\
$-0 /+3$ & $-0 /+5 \%$ & $\%$ \\
15.28 & 13.80 & $\%$ \\
37.12 & 112 & Voltios \\
8.76 & 2.2 & Amperios \\
30.38 & 87.5 & Voltios \\
8.29 & 1.95 & Amperios \\
0.059 & 0.01 & $\% / K$ \\
-0.32 & -30 & $\% / K$ \\
-0.42 & -0.31 & $\% / K$ \\
1.6358 & 1.2281 & $m^{2}$ \\
\hline
\end{tabular}

Tabla 3. Especificaciones técnicas del inversor fotovoltaico Sunny Boy SB 1.5-VL-40

\begin{tabular}{|l|l|l|}
\hline PARAMETRO & VALOR & UNIDAD \\
\hline ENTRADA & 1600 & Watts \\
Potencia Max de entrada DC & 600 & Voltios \\
Tensión Máxima & $160-500$ & Voltios \\
Voltaje DC MMP & 10 & Amperios \\
Corriente de entrada Max & 18 & Amperios \\
Corriente Max de cortocircuito & & \\
SALIDA & 1500 & Watts \\
Potencia Nominal a 230V & 1500 & Watts \\
Potencia aparente & 230 & Voltios \\
Tensión de Red nominal & $220 / 230 / 240$ & Voltios \\
Tensión nominal de CA & 7 & Amperios \\
Corriente de salida Max & $50 / 60$ & Herz \\
Frecuencia de alimentación CA & 97.2 & $\%$ \\
Máxima Eficiencia & &
\end{tabular}


Tabla 4. Especificaciones técnicas del aerogenerador del sistema híbrido

\begin{tabular}{|l|l|l|}
\hline Características & Valor & Unidad \\
\hline Alternador & Trifásico & \\
Tipo & 92 & $\%$ \\
Eficiencia & 3.5 & Kilo \\
Potencia Nominal & 350 & Watts \\
Tensión Nominal & & V AC \\
Turbina & $0-650$ & \\
Gama de velocidad & $115-450$ & $\mathrm{rpm}$ \\
Velocidad de funcionamiento & 2.2 & $\mathrm{rpm}$ \\
Velocidad de arranque & 13 & $\mathrm{~m} / \mathrm{s}$ \\
Protección de Tempestades & $0.39-0.41$ & $\mathrm{~m} / \mathrm{s}$ \\
Coeficiente de potencia & & $\mathrm{m}$ \\
Datos Mecánicos & 3.5 & $\mathrm{~m}^{2}$ \\
Diámetro de rotor & 9.61 & \\
Área de variación & & \\
\end{tabular}

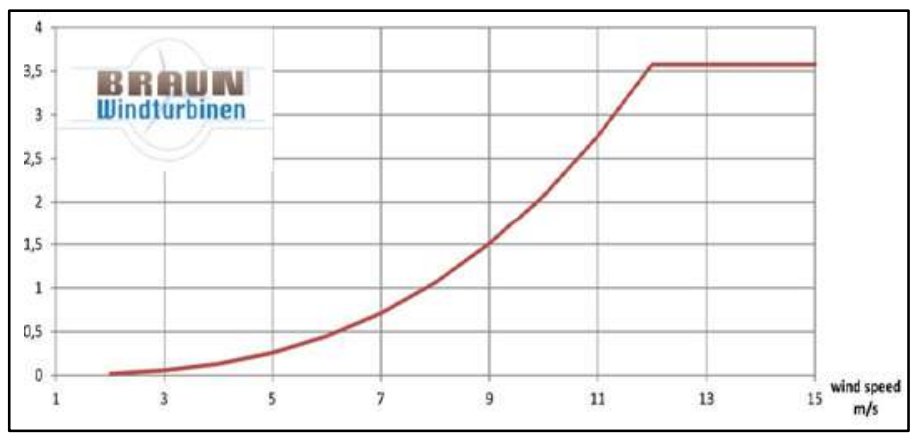

Figura. 3. Curva de potencia del aerogenerador de $3.5 \mathrm{~kW}$ proporcionado por ANTARIS.

\section{METODOLOGIA DE ESTUDIO}


La presente investigación tiene enfoque cuantitativo, de tipo descriptivo, el estudio se realizó en las instalaciones de la escuela de Ingeniería en Energía de la Universidad Nacional del Santa.

Para la recolección de información se empleó la técnica de observación directa a través de reportes de operación para diferentes regímenes de velocidad de viento horizontal obtenidos por un anemómetro de primera clase avanzada 0.5 instalado a 10 metros por encima del suelo; análogamente la radiación proporcionados por un piranómetro de clase A, ubicados estratégicamente en la parte superior del cubo del sistema híbrido eólico solar,

Por medio de una cabina eléctrica donde se encuentran conectados todos los dispositivos para la distribución, la seguridad y la conmutación de las conexiones eléctricas, así como el control y medición registramos cada 5 minutos los parámetros de operación y generación eléctrica iniciales del sistema considerando la variación del clima según la época del año, para su caracterización. El horario de trabajo y recopilación de la información fue de 8:30 hasta 18:30 horas del día.

Asimismo, para representar y procesar los datos se empleó el uso de sistemas informáticos, procesador de datos y hoja electrónica Excel.

\subsection{Viento y generador eólico}

Burton et al. (2001), señalaron que una de las características principales del viento es su gran variabilidad. Sin embargo, se puede describir algunas tendencias en función de la situación geográfica, altitud y topografía.

La potencia aprovechable del viento, conducido por una masa de aire fluido a una velocidad, puede expresarse como:

$$
P_{\text {viento }}=\frac{1}{2} \rho A V^{3}
$$

$\rho=$ Densidad del aire

$A=$ Sección transversal del fluido $\left(\mathrm{m}^{2}\right)$.

$V=$ Velocidad del aire $(\mathrm{m} / \mathrm{s})$. 
A partir de la aplicación de la ecuación de la cantidad de movimiento (Betz, 1927) determinó que la energía máxima teóricamente aprovechable del viento era 16/27 de la energía calculada por la Ec. (1). Entonces se define el coeficiente de potencia $\left(C_{P}\right)$ de un aerogenerador como la fracción de potencia contenida en el viento incidente que es realmente capturada por el aerogenerador. Es una medida de la eficiencia de la máquina.

$$
C_{P}=\frac{P_{\text {A eolica }}}{P_{\text {viento }}}
$$

el par mecánico $(N . m)$ provocado por el viento en el generador viene dado por (Ackermann, 2005):

$$
T_{m}=\frac{1}{2} \rho \pi R^{3} V^{2} \frac{C_{p}}{\lambda}
$$

$R(m)$ el radio de la pala, $\lambda$ velocidad de punta de pala.

$$
\lambda=\frac{R \omega}{V}
$$

donde $\omega(\mathrm{rad} / \mathrm{s})$ es la velocidad angular.

\subsection{Modelo del módulo fotovoltaico}

Factor de forma. Es un factor que sirve como dato para hallar la eficiencia de una célula o módulo fotovoltaico. Es la relación entre la potencia máxima teórica y la potencia real medido en el momento solar.

$$
F F=\frac{V_{m p p} I_{m p p}}{V_{o c} I_{s c}}
$$

La eficiencia de conversión de un módulo fotovoltaico es el valor de la energía eléctrica aprovechada del total de la energía solar impactada sobre la superficie del módulo.

$$
e=\frac{P_{G E F}}{G A_{C}} 100
$$


siendo $G\left(1000 \mathrm{~W} / \mathrm{m}^{2}\right)$ la irradiancia a condiciones $\mathrm{CEM}, P_{G E F}(W)$ la potencia de generación eléctrica real fotovoltaica y $A_{c}\left(\mathrm{~m}^{2}\right)$ el área del módulo fotovoltaico.

El rendimiento de generación de energía eléctrica es el valor del cociente de la potencia de generación eléctrica real y la potencia nominal $\left(P_{n}\right)$ de los módulos fotovoltaicos.

$$
n=\frac{P_{G E F}}{P_{n}} 100
$$

\subsection{Modelo del sistema híbrido eólico fotovoltaico}

El rendimiento de generación de energía eléctrica del sistema híbrido este dado por el cociente de la suma de la potencia generada por los generadores fotovoltaicos y la potencia generada por la turbina eólica, y la potencia nominal del sistema híbrido.

$$
n=\frac{P_{\text {A eolica }}+P_{\text {fotovol }}}{P_{\text {nom.hibrido }}}
$$

Para calcular la eficiencia de conversión de energía del sistema híbrido está dado por el cociente de la suma de la potencia generada real del aerogenerador y los módulos fotovoltaicos, y la suma de la potencia total de la energía solar impactada en los generadores fotovoltaicos y la potencia del viento del aerogenerador.

$$
e=\frac{P_{\text {A eolica }}+P_{\text {fotovol }}}{P_{\text {viento }}+I A_{c}}
$$

Siendo $I\left(w / m^{2}\right)$ la irradiancia medida a tiempo real y $A_{c}\left(m^{2}\right)$ superficie que cubren los módulos fotovoltaicos.

\section{RESULTADOS}

\section{Potencia generada}




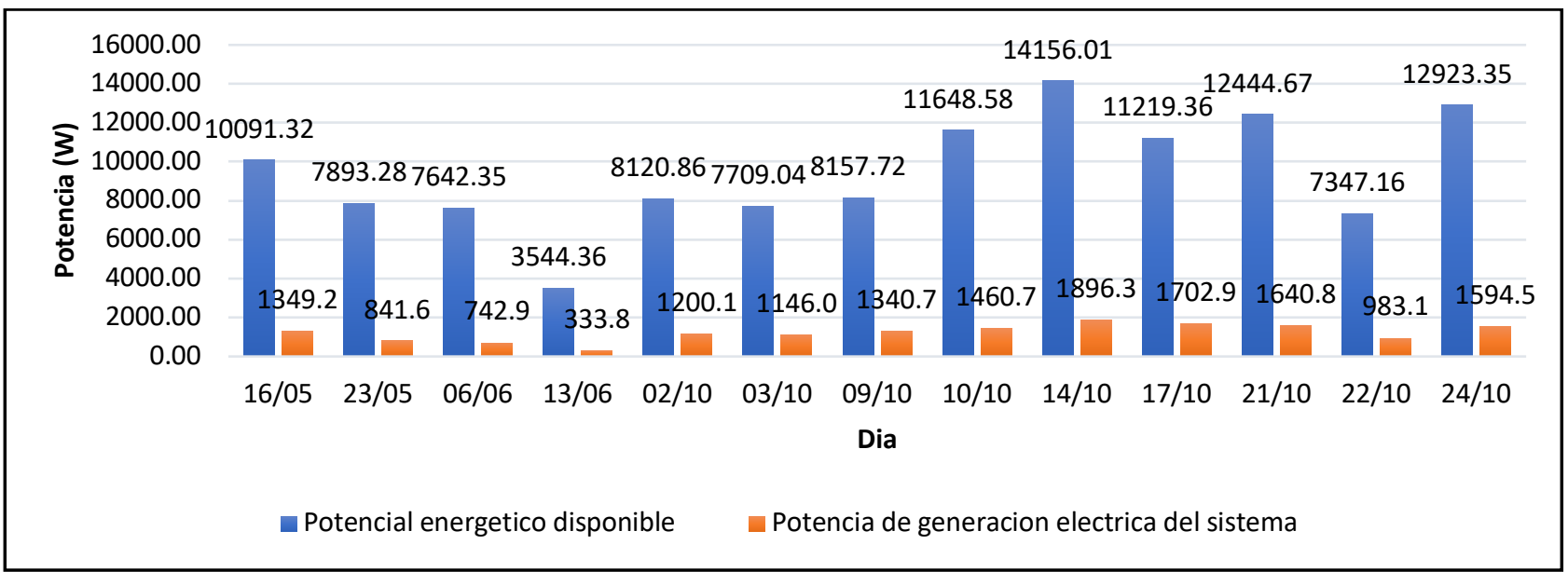

Figura. 4. Relación entre la potencia diaria recibida por el sistema hibrido eólicofotovoltaico, y la potencia de generación eléctrica del sistema.

En la Fig. 4. Para el periodo de trabajo se puede observar la relación de potencias promedio recibida por el sistema hibrido eólico fotovoltaico, tanto de los paneles fotovoltaicos dada por la irradiancia solar, y del aerogenerador dada por la velocidad de viento, así como la potencia de generación eléctrica del sistema. La gran diferencia entre la energía entregada por el sol y el viento, y la energía generada por el sistema híbrido, se debe a la eficiencia nominal de los paneles fotovoltaicos que van 15.28\% (Silicio policristalino) y $13.8 \%$ (Película delgada CSI) así como el coeficiente de potencia de la turbina eólica de 0.41. La potencia generada se ve afectada por el incremento de la temperatura del módulo, la suciedad de los paneles fotovoltaicos, y el efecto sombra que hace el aerogenerador a los paneles fotovoltaicos y la ausencia de velocidad de viento para el funcionamiento de la turbina eólica. 


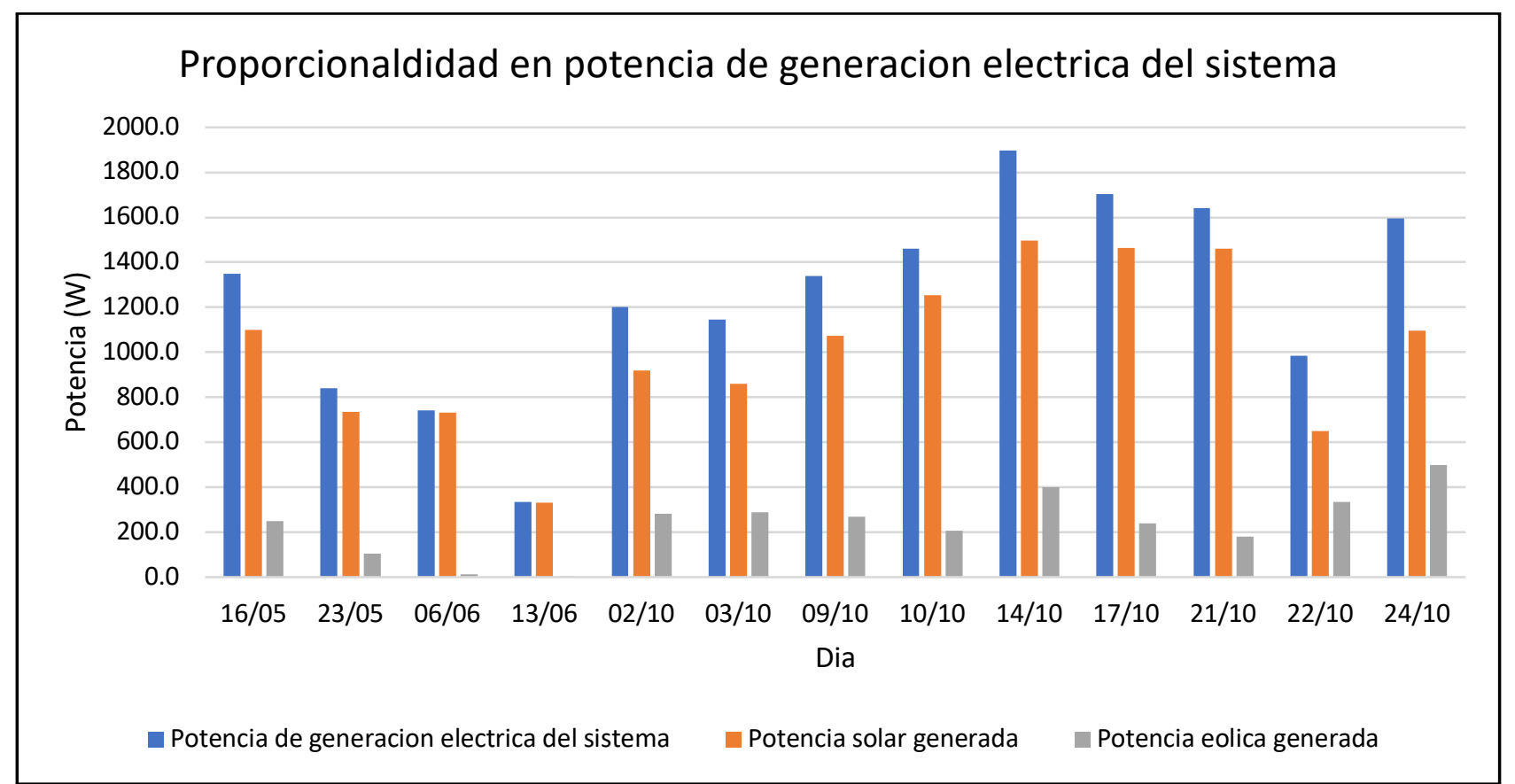

Figura. 5. Proporcionalidad en función al orden de aportación de potencia de los paneles fotovoltaicos y el aerogenerador al sistema hibrido.

En la Fig. 5. Se puede claramente apreciar que el principal aportante de potencia para el sistema son los paneles fotovoltaicos. Este se encuentra claramente con ventaja en su funcionamiento, ya que solo requiere la presencia de irradiación solar, por otro lado el aerogenerador para su arranque necesita una velocidad de viento mínimo de $2.2 \mathrm{~m} / \mathrm{s}$ y para alcanzar un valor máximo de potencia generada necesita una velocidad de viento superior a $11.5 \mathrm{~m} / \mathrm{s}$ para generar una potencia nominal de $3500 \mathrm{~W}$, y el promedio de velocidades de viento estudiados alcanzan un valor mínimo de $1.20 \mathrm{~m} / \mathrm{s}$ y máximo de $5.41 \mathrm{~m} / \mathrm{s}$ que con el coeficiente de potencia del aerogenerador disminuye considerablemente.

Tabla 5. Irradiación y velocidad promedio medido en el sistema hibrido eólico fotovoltaico según fecha indicada.

\begin{tabular}{|l|l|l|}
\hline Dia medido & Irradiación $\left(\boldsymbol{w} / \boldsymbol{m}^{\mathbf{2}}\right)$ & Velocidad $(\boldsymbol{m} / \mathbf{s})$ \\
\hline 16 de mayo & 527.26 & 3.84 \\
\hline 23 de mayo & 424.27 & 2.82 \\
\hline 6 de junio & 422.90 & 1.33 \\
\hline
\end{tabular}




\begin{tabular}{|l|l|l|}
\hline 13 de junio & 196.60 & 1.20 \\
\hline 2 de octubre & 435.52 & 4.33 \\
\hline 3 de octubre & 412.33 & 4.38 \\
\hline 9 de octubre & 573.94 & 4.23 \\
\hline 10 de octubre & 635.73 & 4.16 \\
\hline 14 de octubre & 764.27 & 4.94 \\
\hline 17 de octubre & 610.07 & 4.16 \\
\hline 21 de octubre & 681.37 & 4.00 \\
\hline 22 de octubre & 389.71 & 4.85 \\
\hline 24 de octubre & 690.39 & 5.41 \\
\hline
\end{tabular}

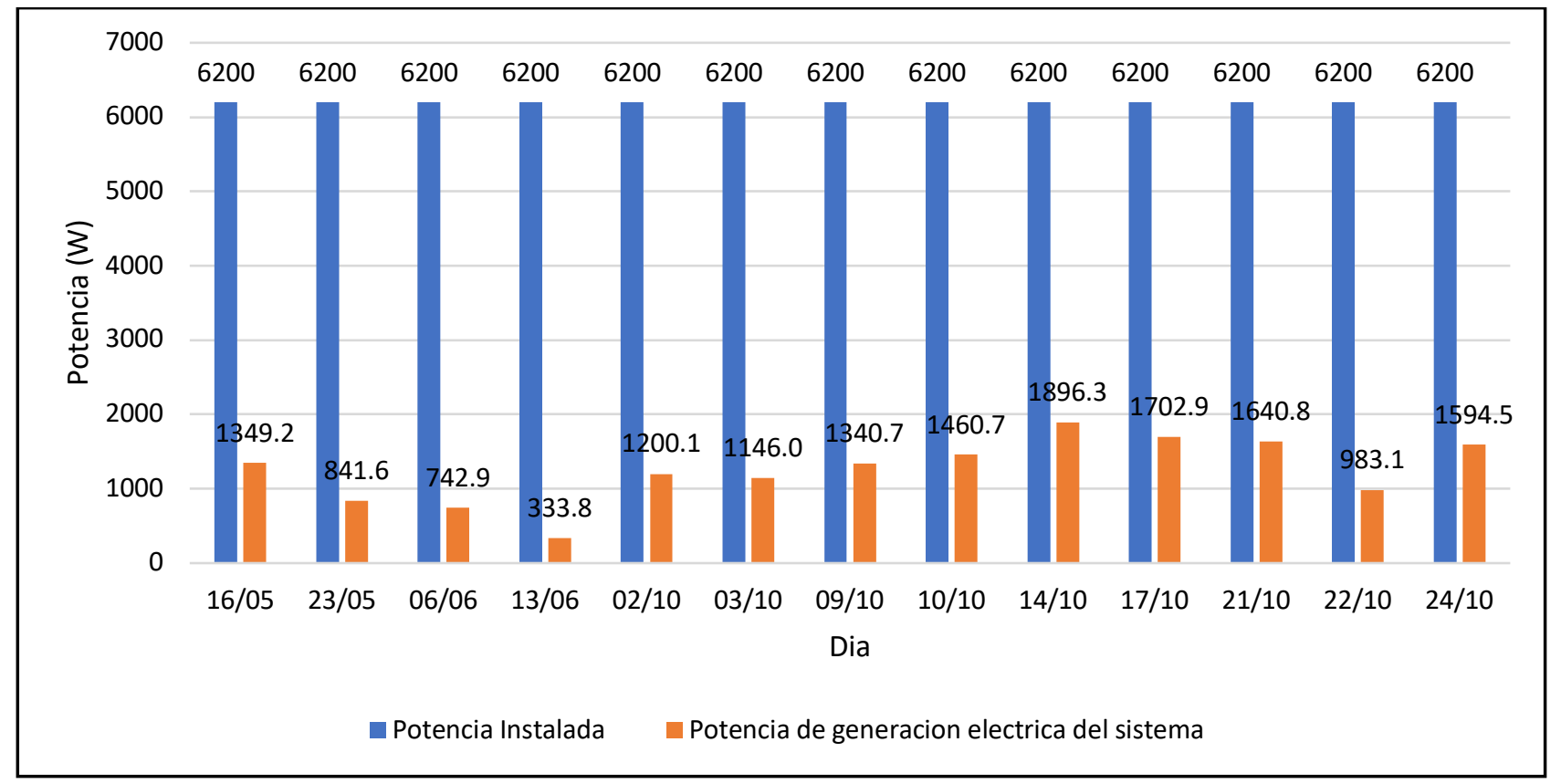

Figura. 6. Comparación entre la potencia instalada y la potencia de generación eléctrica del sistema híbrido.

La gráfica presentada en la Fig.6. nos muestra la potencia instalada que asciende a los 6 $200 \mathrm{~W}$ frente a la potencia generada que como máximo alcanzó el valor de $1896 \mathrm{~W}$. La explicación de este comportamiento se debe principalmente a dos factores: La insuficiencia de los recursos estudiados y la labor del inversor de batería. Los valores recopilados en los meses fríos muestran un comportamiento referente a la variabilidad incontrolable de la fuente mientras que, en los meses calientes, el inversor de batería 
cumple con la función no sobrecargar el acumulador pues al no contar con consumidores finales, la única salida viable es el moderar la generación conteniendo la labor de los paneles fotovoltaicos esto se traduce en el desaprovechamiento de potencia disponible y accesible.

Rendimiento de generación de energía eléctrica y eficiencia de conversión del sistema hibrido eólico fotovoltaico.

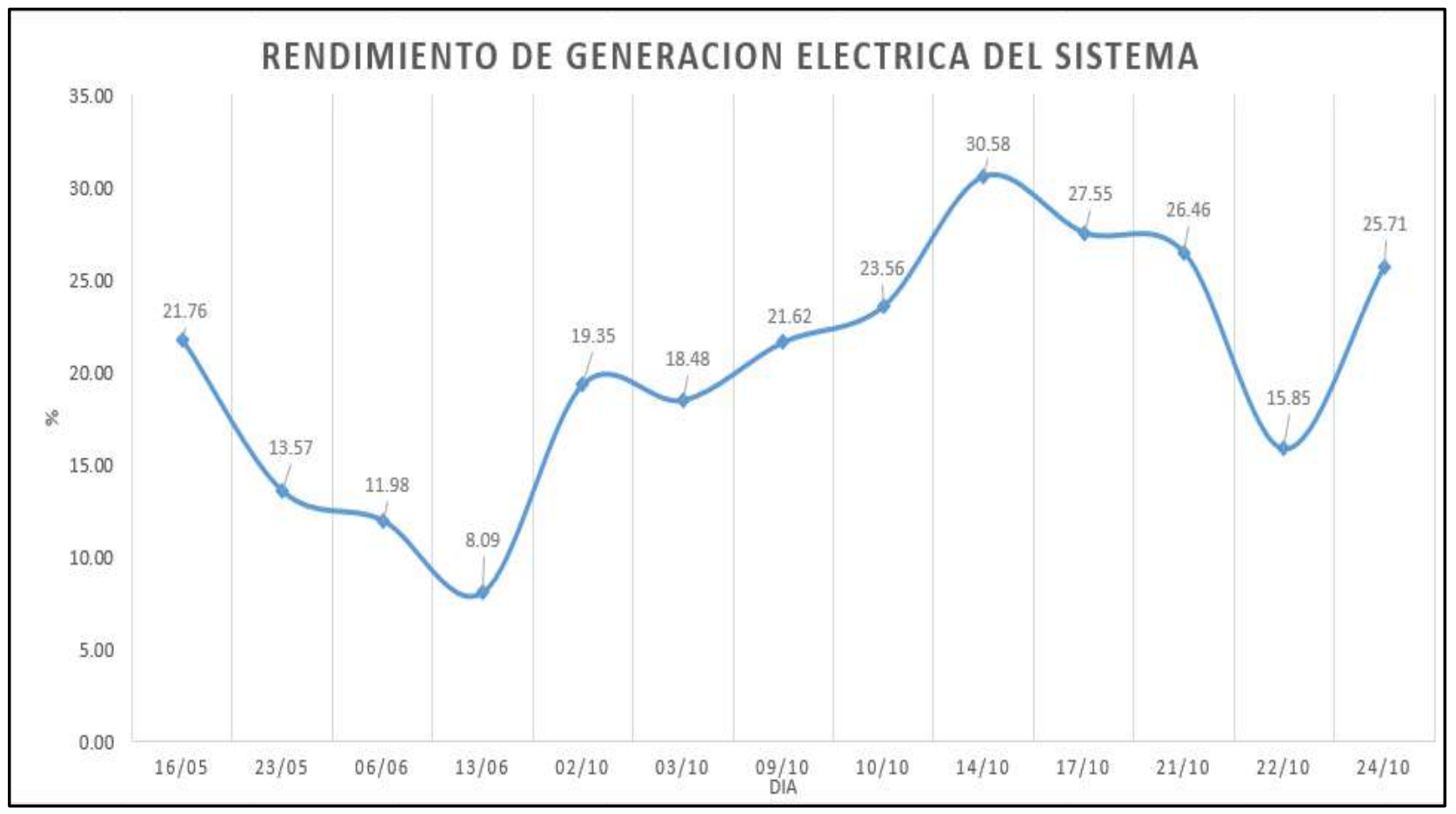

Figura. 7. Gráfica del rendimiento de generación de energía eléctrica del sistema hibrido eólico fotovoltaico. 


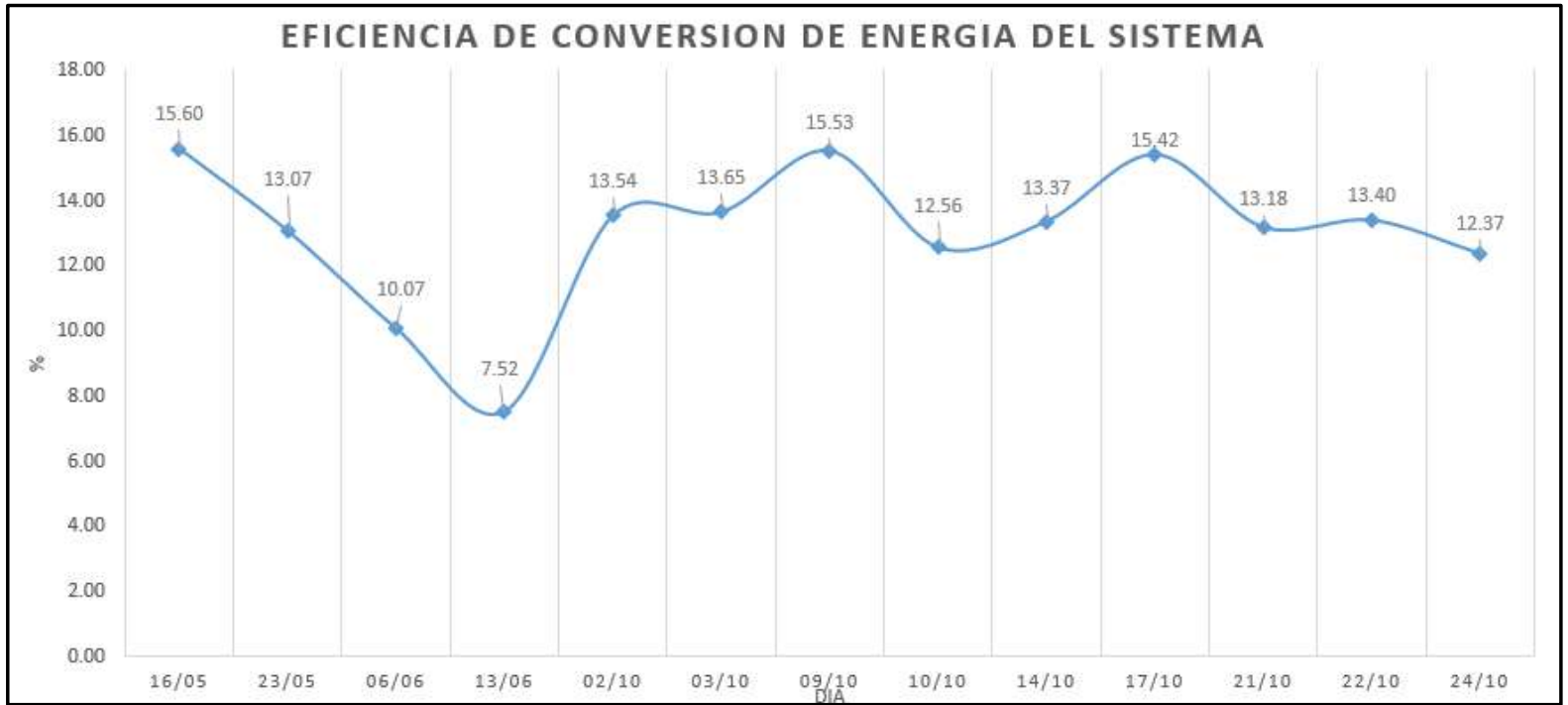

Figura. 8. Gráfica de la eficiencia de conversión de energía del sistema hibrido eólico fotovoltaico.

En la Fig. 7. Observamos la variación del rendimiento de generación de energía eléctrica total alcanzando un valor máximo igual a 30.58\% y en la Fig. 8. Observamos que la eficiencia de conversión de energía del sistema hibrido eólico fotovoltaico alcanzando un valor máximo de $15.42 \%$ este valor va disminuyendo en el mes de mayo y junio hasta valores de rendimiento de $8.09 \%$ y de eficiencia $7.52 \%$ estos valores se deben a que entran a fechas del peor mes donde el recurso eólico y solar disminuye.

\section{CONCLUSIONES}

Según la evaluación y caracterización realizada al sistema hibrido eólico fotovoltaico, instalado en la Loza Solar de la Universidad Nacional del Santa, con una potencia nominal de $6,2 \mathrm{~kW}$, se concluye que, bajo las condiciones meteorológicas de la ciudad de Nuevo Chimbote, la potencia promedio máxima generada fue de $1896.3 \mathrm{~kW}$.

El rendimiento de generación de energía eléctrica del Sistema hibrido eólico fotovoltaico alcanza un valor promedio de aproximadamente $30 \%$ para condiciones del mes de octubre donde empieza las estaciones de primavera y $8 \%$ para condiciones del mes de junio donde empiezan las épocas donde existe poca radiación y velocidad de viento, lo cual nos indica que la potencia generada está por debajo de los estándares esperados con energías renovables para autoconsumo y posible aprovechamiento según su demanda; esto se debe a la acción de sistemas de protección incorporados de la instalación. Con la demanda 
eléctrica creciente en el mercado nacional, se presenta este caso como antecedente de lo importante en el aprovechamiento de un sistema híbrido eólico solar con el fin de obtener un desarrollo sostenido.

Dadas las condiciones meteorológicas de la ciudad de Nuevo Chimbote para los días evaluados existe conjuntamente la presencia del recurso eólico y solar para condiciones donde el rendimiento del sistema hibrido alcanza valores máximos, inversamente proporcional se da cuando el rendimiento llega a valores mínimos, es en estas fechas donde en necesaria un aporte auxiliar como un generador eléctrico o una conexión a la red eléctrica pública.

\section{BIBLIOGRAFIA}

Ackerman, T. 2005. Wind Power and Power Systems. John Wiley \& Sons.

Bravo, C. Contreras, A., 2017. Evaluación del funcionamiento de un sistema fotovoltaico de $3.3 \mathrm{KW}$ interconectado a la red eléctrica del CERT-UNJBG, Tacna: octubre 2016 octubre 2017, Huaraz, 13 -17.11.2017.

Burton, T. Sharpe, D. Jenkins, N. Bossanyi, E. 2001. Wind Energy handbook. John Wiley \& Sons.

Candia, R. 2012. Metodología para cálculo de curva de potencia de turbina eólica de eje vertical. Tesis para el grado de Magister. Pontifica Universidad Católica de Chile.

Carrera, M, Diéguez, E. 2021. Estimación del ahorro energético y alternativas de energía eléctrica en pymes turísticas del cantón Junín. Revista Internacional de Gestión, Innovación y Sostenibilidad Turística, Vol. 1, N¹, 36-51.

Díaz, C. Carmona, R. 2017. Instalaciones solares fotovoltaicas. Componentes de una instalación fotovoltaica, pág.7-18.

Diaz, L. 2010. Análisis Energético de un Sistema Híbrido eólico-fotovoltaico en el sector típico IV utilizando el Método de Series Sintéticas, Tesis para el grado de ingeniero. Universidad Nacional del Centro.

González, R. 2019. Propuesta de un Sistema Híbrido Eólico - Fotovoltaico para el Hotel Santa Clara Libre. Universidad Central "Marta Abreu" de las Villas.

Hervas, J. Moscoso, M. 2015. Análisis del potencial eólico y solar para la implementación de un sistema híbrido de generación eléctrica en base al uso de energías alternativas en el páramo Chalupas, periodo 2015. Tesis para el grado de Ingeniero. Universidad Técnica de Cotopaxi. 
Méndez, F. 2010. Análisis y técnicas para la implementación de un sistema híbrido solareólico. Tesis para el grado de ingeniero. Universidad Austral de Chile.

Mendosa, L. Palomino, J. e Higueras, J., 2017. Caracterización de paneles solares a sol real, XXII Simposio Peruano de Energía Solar y del Ambiente (XXIV-SPES), Huaraz, $13-17.11 .2017$

Mitaki, M. Santos, M. Armenta, C. 2012. Modelado y Simulación de un Sistema Conjunto de Energía Solar y Eólica para Analizar su Dependencia de la Red Eléctrica. Revista Iberoamericana de Automática e Informática Industrial RIAI

OSINERMING, 2017. La industria de la energía renovable en el Perú: 10 años de contribuciones a la mitigación del cambio climático. Primera edición.

Pallabazer, R. 2004. Previsional estimation of the energy output of windgenerators, Renewable Energy: 29, 413-420.

Pinto, M. 2012. Fundamentos de la energía eólica. EDEL.

Ramos, H., Luna, R. 2014. Diseño de un sistema fotovoltaico integrado a la red para el área de estacionamiento de la universidad tecnológica de Salamanca. Tesis de Maestría. Centro de investigación en materiales avanzados.

Rojas. A. 2012. Evaluación del Recurso Eólico de la UNS para proyección de un bosque eólico de autogeneración. Tesis de Maestría. Universidad Nacional del Santa.

Ruiz, J. 2019. Dimensionamiento de un sistema hibrido de energía renovable solarbiomasa para satisfacer la demanda eléctrica del centro poblado el afluente-Rioja-San Martín. Universidad Nacional "Pedro Ruiz Gallo".

Villamil, J., Villamil, M. 2019. Aplicación y utilización de fuentes de energías combinadas - solar y eólica-, como alternativa de autonomía eléctrica y generación de ingresos por la capacidad residual ingresada al sistema de suministro de energía convencional. Universidad EAN.

Villena, M. Serrano, V. Montero, M. y Zutara, F., 2017. Caracterización de un módulo fotovoltaico de silicio amorfo y simulación con GNU OCTAVE, XXII Simposio Peruano de Energía Solar y del Ambiente (XXIV- SPES), Huaraz, 13 -17.11.2017. 